\title{
Spectroscopy of ${ }^{9} \mathrm{C}$ via resonance scattering of protons on ${ }^{8} \mathrm{~B}$.
}

\author{
G.V. Rogachev, ${ }^{1}$, 困 J.J. Kolata, ${ }^{2}$ A.S. Volya, ${ }^{1}$ F.D. Becchetti, ${ }^{3}$ Y. Chen,${ }^{3}$ P.A. DeYoung, ${ }^{4}$ and J. Lupton ${ }^{3}$ \\ ${ }^{1}$ Department of Physics, Florida State University, Tallahassee, FL, 32306 \\ ${ }^{2}$ Department of Physics, University of Notre Dame, Notre Dame, IN, 46556 \\ ${ }^{3}$ Department of Physics and Astronomy, University of Michigan, Ann Arbor, MI, 48109 \\ ${ }^{4}$ Department of Physics, Hope College, Holland, MI, 49422
}

\begin{abstract}
The structure of the neutron-deficient ${ }^{9} \mathrm{C}$ isotope was studied via elastic scattering of radioactive ${ }^{8} \mathrm{~B}$ on protons. An excitation function for resonance elastic scattering was measured in the energy range from 0.5 to $3.2 \mathrm{MeV}$ in the center-of-momentum system. A new excited state in ${ }^{9} \mathrm{C}$ was observed at an excitation energy of $3.6 \mathrm{MeV}$. An R-matrix analysis indicates spin-parity $5 / 2^{-}$for the new state. The results of this experiment are compared with Continuum Shell Model calculations.
\end{abstract}

\section{INTRODUCTION}

Light nuclei play a central role in nuclear physics since they are the simplest cases where nuclear many-body dynamics can be understood and explored. This special role is reflected in the number of different theoretical approaches that exist, and overlap, in addressing the structure of light nuclei. The techniques range from full $a b$ initio methods, such as the Green's Function Monte Carlo method [1] or the Large Basis No-Core Shell Model method [2], in which the properties of light nuclei are computed starting from bare nucleon-nucleon interactions, to the traditional shell-model approach with renormalized or phenomenologically-determined interactions, to cluster models. Light nuclei also provide an important arena for exploring physics on the interface between structure and reactions. The dawn of modern structurereaction unification is marked by several recent theoretical developments: the Gamow Shell Model (GSM) [3] and Continuum Shell Model (CSM) 4] in particular. Realistic tests of these models constitute an important step both in theoretical developments in the description of the properties of exotic nuclei and in furthering our understanding of the role that exotic resonances play in nuclear astrophysics.

In the analysis of the experimental study reported below we use the CSM approach which permits calculation of resonance parameters and cross sections, thus allowing a direct comparison with experimental data. A comparison with older techniques such as a traditional R-matrix analysis with shell-model spectroscopic factors highlights their limitations and gives a valuable insight into physics on the reaction/structure borderline encompassed by the advanced CSM technique.

The focus of this work is the structure of an exotic neutron-deficient dripline isotope of carbon, ${ }^{9} \mathrm{C}$, which has a halflife of $126.5 \mathrm{~ms}$ and a binding energy of 1.3 $\mathrm{MeV}$. Spectroscopic information about this nucleus is scarce. The ground state of ${ }^{9} \mathrm{C}$ was first identified in the ${ }^{12} \mathrm{C}\left({ }^{3} \mathrm{He},{ }^{6} \mathrm{He}\right)$ reaction by Cerny, et al. 5] in 1964 .

\footnotetext{
*Electronic address: grogache@fsu.edu
}

Ten years later, the first excited state of ${ }^{9} \mathrm{C}$ was observed at $2.2 \mathrm{MeV}$ using the same reaction [6]. More recently, ${ }^{12} \mathrm{C}\left({ }^{3} \mathrm{He},{ }^{6} \mathrm{He}\right)$ was yet again studied in Ref. 7]. The authors of this work claim to have seen another excited state at about $3.3 \mathrm{MeV}$. However, the complicated nature of the reaction mechanism did not allow for a reliable spin-parity assignment. Instead, indirect arguments were given favoring spin-parity of $5 / 2^{+}$for this level 7 ].

Modern experimental techniques allow for the use of beams of radioactive nuclei to populate states in exotic isotopes by means of simple reactions such as one-nucleon transfer or resonance elastic scattering. As a result, more reliable identification on the properties of the observed states can now be obtained. In the present work, excited states in ${ }^{9} \mathrm{C}$ are populated via resonance elastic scattering of radioactive ${ }^{8} \mathrm{~B}$ on protons. The main goal of this work was to identify the excited states in ${ }^{9} \mathrm{C}$ and compare the properties of these states with theoretical predictions.

\section{EXPERIMENT}

The experiment was performed using the TwinSol radioactive nuclear beam (RNB) facility at the University of Notre Dame [8]. A $2.5 \mathrm{~cm}$ long gas target containing 1 atm of ${ }^{3} \mathrm{He}$ was bombarded by a nanosecond-bunched primary ${ }^{6} \mathrm{Li}$ beam at an energy of $39.0 \mathrm{MeV}$ and intensity of 200 electrical $\mathrm{nA}$. The two-proton pickup reaction ${ }^{3} \mathrm{He}\left({ }^{6} \mathrm{Li},{ }^{8} \mathrm{~B}\right) \mathrm{n}$ was used to produce the ${ }^{8} \mathrm{~B}$ ions. The entrance and exit windows of the gas cell consisted of 2.0 $\mu \mathrm{m}$ Havar foils. The secondary ${ }^{8} \mathrm{~B}$ beam was momentum selected and transported through the two superconducting solenoids, which focused it into a $5 \mathrm{~mm}$ spot on a $9.0 \mathrm{mg} / \mathrm{cm}^{2}$ plastic $\left(\mathrm{CH}_{2}\right)$ target. The laboratory energy of the ${ }^{8} \mathrm{~B}$ beam at the secondary target position was $29 \mathrm{MeV}$, with a resolution of $0.7 \mathrm{MeV}$ full width at half maximum (FWHM) and an intensity of up to $10^{4}$ particles per second. The energy spread was due to a combination of the kinematic shift in the production reaction plus energy-loss straggling in the gas-cell windows. Contaminant ions which had the same magnetic rigidity as $29 \mathrm{MeV}{ }^{8} \mathrm{~B}$ also were present in the beam, but they could be identified using the time-of-flight (TOF) technique. The TOF of the particles was obtained from the 


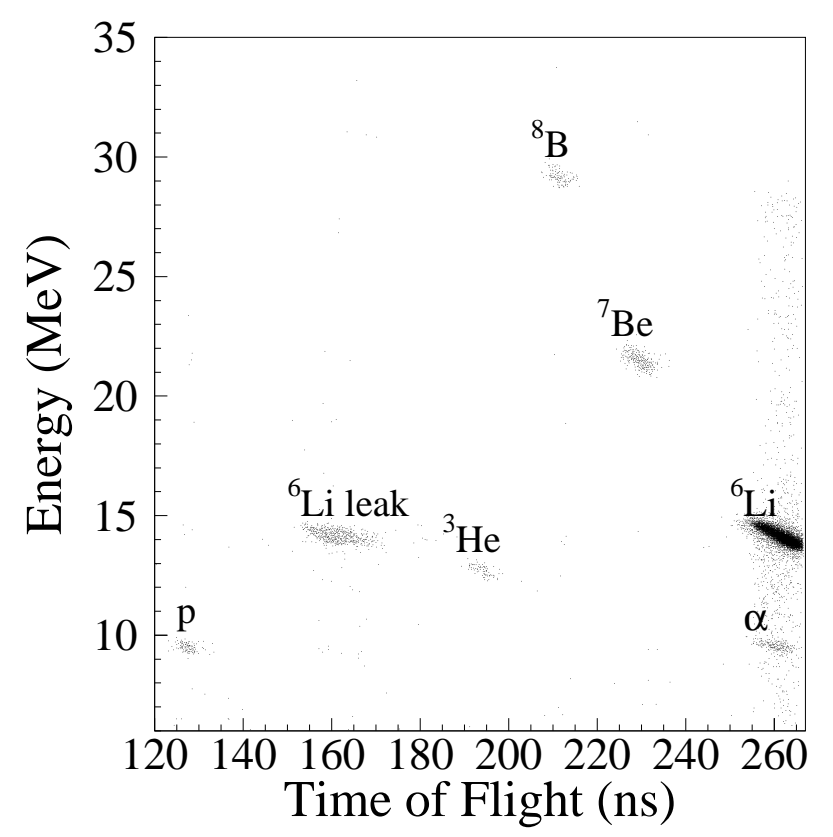

Figure 1: The total energy of a beam ion vs. its time of flight measured with the Au target by a Si $\Delta \mathrm{E}-\mathrm{E}$ telescope placed at $7.7^{\circ}$. The start signal was taken from the $\mathrm{E}$ detector of the telescope and the stop signal came from the beam buncher.

time difference between the occurrence of an E signal in a detector telescope and the RF timing pulse from the beam buncher. The time resolution of better than $5 \mathrm{~ns}$ (FWHM) was adequate to cleanly separate ${ }^{8} \mathrm{~B}$ from all other ions (except for some direct protons as will be discussed further in the text). This is illustrated in Fig. 1] which was obtained with a $1.0 \mathrm{mg} / \mathrm{cm}^{2}$ Au target and a Si $\Delta$ E-E telescope placed at $7.7^{\circ}$ with respect to the beam axis. The intensity of the beam during the experiment was determined from the ratio of the ${ }^{8} \mathrm{~B}$ ions to the integrated charge of the primary ${ }^{6} \mathrm{Li}$ beam collected in the TwinSol Faraday cup. This ratio was measured by placing the Si $\Delta \mathrm{E}-\mathrm{E}$ telescope directly at the target position.

The thick-target inverse-kinematics technique [9] was used to measure the excitation function of ${ }^{8} \mathrm{~B}+\mathrm{p}$ resonance elastic scattering. The plastic target was thick enough $\left(9.0 \mathrm{mg} / \mathrm{cm}^{2}\right)$ to stop the $29 \mathrm{MeV}^{8} \mathrm{~B}$ ions. The recoil protons from back-angle elastic scattering in this target lose only a small amount of energy in traversing the foil and emerge from it with sufficient energy to be detected. Note that the lowest-energy protons, from the scattering of ${ }^{8} \mathrm{~B}$ ions near the end of their range, encounter the least amount of material before leaving the target. In this manner, an excitation function for elastic scattering down to very low energies can be measured with high efficiency and good energy resolution. The recoil protons were detected with two telescopes consisting of 19.5 and $19.2 \mu \mathrm{m}$ Si $\Delta \mathrm{E}$ detectors, backed by $1000 \mu \mathrm{m}$ $\mathrm{Si} \mathrm{E}$ detectors. The active area of the $\Delta \mathrm{E}$ detectors was
$450 \mathrm{~mm}^{2}$, and that of the E detectors was $600 \mathrm{~mm}^{2}$. Each telescope had a circular collimator with a diameter of 18 $\mathrm{mm}$ that subtended a solid angle of $11.6 \mathrm{msr}$. They were placed on either side of the beam at $7.7^{\circ}$ with respect to the beam axis. It would have been preferable to place a telescope at $0^{\circ}$ to the beam but the light-ion contamination (Fig. 1) produced a count rate in this position that was unacceptable since these ions penetrated the target and directly entered the telescope.

It was verified that the recoil proton TOF signal was only slightly shifted in time relative to ${ }^{8} \mathrm{~B}$ and was stable during the course of the experiment so that the separation from elastic scattering of contaminant ions was excellent. Nevertheless, a background associated with direct protons scattered by the plastic target was still present in the proton spectrum measured by the telescopes, even after the TOF gate. The origin of this proton background is the unfortunate coincidence between the difference of the actual flight times of ${ }^{8} \mathrm{~B}$ and protons from the primary to the secondary target $(\sim 80 \mathrm{~ns})$ and the half-period of the buncher (100 ns). Because of this coincidence, a small fraction of the direct protons from the tail of the proton time distribution overlaped with the timing of the ${ }^{8} \mathrm{~B}$ ions from the "previous" bunch. This background was reduced by two orders of magnitude after a pulse selection was introduced (the efficiency of pulse selection was $99 \%$ ), making the period between beam bunches 200 ns. Still, some direct protons from the tail of the proton time distribution leaked into the gate, as shown in Fig. 2 which represents the raw proton spectrum measured by one of the telescopes gated by the ${ }^{8} \mathrm{~B}$ TOF. This background was eliminated by subtracting the spectrum of protons gated on timing in the region between ${ }^{8} \mathrm{~B}$ and ${ }^{7} \mathrm{Be}$ (dashed line in Fig. 2) from the spectrum of protons associated with ${ }^{8} \mathrm{~B}$.

Another source of background are protons produced in the interaction of the ${ }^{8} \mathrm{~B}$ with carbon in the plastic target. These protons have exactly the same timing as the recoil protons from elastic scattering of ${ }^{8} \mathrm{~B}$ on hydrogen. The spectrum of this process, measured using a $15.8 \mathrm{mg} / \mathrm{cm}^{2}$ thick carbon target is shown as the shaded histogram in Fig. 3] together with the total spectrum of protons from the plastic target measured by both telescopes. The thickness of the carbon target was adjusted to match the thickness of the plastic target in terms of energy loss. The "carbon" background spectrum was scaled by a factor of 1.98 to reflect the difference between the integrated number of ${ }^{8} \mathrm{~B}$ ions accumulated during the main and background runs, adjusted to account for the different number of carbon atoms per $\mathrm{cm}^{2}$ for the same energy loss in the carbon and plastic targets. The polynomial fit to the "carbon" background (shown as a solid line in Fig. 31) was then subtracted from the spectrum of protons obtained with the plastic target. 


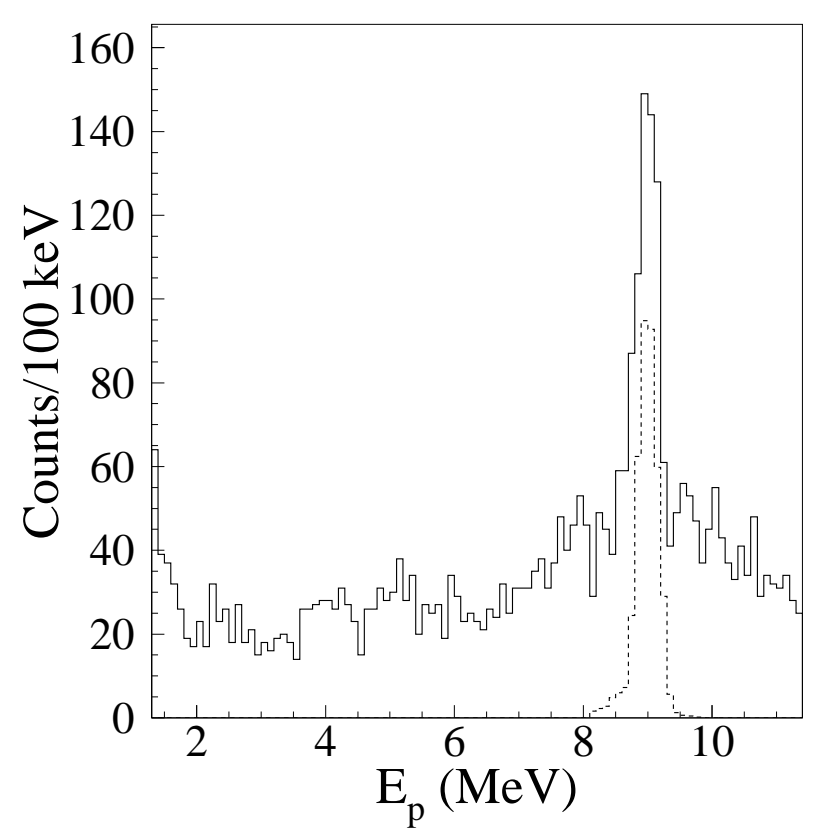

Figure 2: The raw spectrum of protons measured by one of the telescopes and gated on ${ }^{8} \mathrm{~B}$ TOF. The sharp peak at 9 $\mathrm{MeV}$ is due to direct protons scattered by the plastic target. The dashed line is a spectrum of protons produced by gating on the TOF between ${ }^{8} \mathrm{~B}$ and ${ }^{7} \mathrm{Be}$.

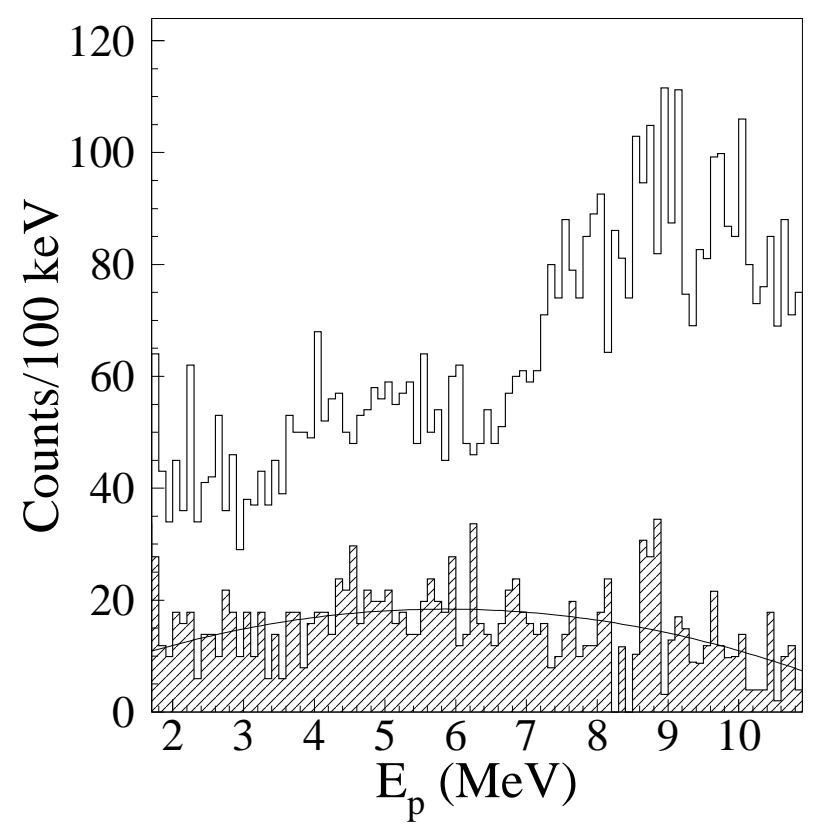

Figure 3: The laboratory spectrum of protons from both telescopes measured with the plastic target. The background associated with the direct protons from the beam has been subtracted. The normalized proton background from the interaction of ${ }^{8} \mathrm{~B}$ with carbon in the plastic target is shown as the shaded histogram. The solid line represents the polynomial fit to the carbon background which was used for subtraction of this background from the total spectrum.

\section{STRUCTURE OF ${ }^{9} \mathrm{C}$ IN THE CONTINUUM SHELL MODEL.}

The continuum of reaction states is an inseparable part of the ${ }^{9} \mathrm{C}$ structure. Thus, for the analysis and interpretation of the experimental results we used the Continuum Shell Model (CSM) developed in Ref. [4]. The traditional shell-model (SM) Hamiltonian that describes the internal evolution of the system is supplemented here with the continuum of reaction states. For the model Hamiltonian we use an s-p-sd-pf valence space with the WBP interaction 10]. The Hamiltonian for protons in the continuum is given by a Woods-Saxon potential. The parameters of this potential were taken from Ref. [11]. The experimental value of the reduced mass of the ${ }^{8} \mathrm{~B}+p$ system was used. For the discussion here, the relevant set of channels includes the $2^{+}$ground state and $1^{+}$first excited state of ${ }^{8} \mathrm{~B}$. These states are well reproduced by the above WBP interaction, but the CSM reaction calculations were performed using the actual experimentally-known $Q$-values. The results from the CSM calculation are presented in Table I This table also includes the spectroscopic factors computed for the corresponding channels.

The spectroscopic factors are defined here in a conventional way as overlap integrals $\left\langle\psi_{S M} \mid \psi_{8_{B}} \otimes \psi_{p}\right\rangle$, neglecting the effects of the continuum. Using these spectroscopic factors, and the decay widths $\Gamma_{W S}$ resulting from the potential-model calculation, a perturbative approach can also be used to estimate the decay widths. The results of the perturbative calculation (not shown) are extremely close to those from the full CSM calculation given in the table. This agreement highlights the fact that CSM, by construction, extends the traditional SM approach yet yields identical results for bound states and almost unchanged results for isolated narrow resonances as the conventional SM. To assess the role of the continuum beyond the lowest order in perturbation, in the third column we show the eigenstate reorientation angle $\Theta$, defined as $\cos \Theta=\left\langle\psi_{S M} \mid \psi_{C S M}\right\rangle$. This angle reflects the relative change in the wave function due to the presence of the continuum. For bound states, the angle is zero confirming that SM and CSM eigenstates are identical. The second $3 / 2^{-}$shows a noticeable deviation which can be explained by its interaction through the continuum with a higher-lying $3 / 2^{-}$state at $6.27 \mathrm{MeV}$ in excitation having a width (determined by the CSM) of 3.6 MeV. Despite their almost 2.6 MeV separation, the large widths allow these two $3 / 2^{-}$states to overlap leading to the noticeable changes in the structure. The case of the $3 / 2^{+}$state is similar.

It is well established that parametrization of a broad resonance by the energy of its centroid and its width is particularly ambiguous in the case of broad and/or overlapping resonances, as well as those close to thresholds. The above case of the interference between $3 / 2^{-}$states is a good example of this phenomenon. The interference effects, while having a moderate effect on the parameters of the resonances, nonetheless result in noticeable 
changes in the cross sections. In Fig. 8 (discussed below), the CSM was used to calculated the inelastic ${ }^{8} \mathrm{~B}+\mathrm{p}$ cross section, which at low energies is dominated by the above mentioned pair of $3 / 2^{-}$states. While the appearance of the peaks is very consistent with a parametrization by energy and width, the off-peak behavior reflects the significant role of interference. The comparison with Rmatrix calculations that include only a single resonance is indicative of this phenomenon. (Further comparison of cross section curves and theoretical methods is interesting but remains beyond the scope of this paper.)

Given the large variety of available shell model interactions, we estimate the theoretical uncertainty in our results by conducting another calculation with a different interaction. The "PWT" interaction [10] was used for the results given in Table II This older interaction includes only the p-shell. The Woods-Saxon Hamiltonian describing the continuum remained unchanged in this calculation.

\section{INELASTIC BACKGROUND IN THE PROTON SPECTRUM.}

The excitation function for the resonance scattering of ${ }^{8} \mathrm{~B}$ on protons is shown in Fig. 4 The background associated with carbon in the target and with direct protons has been subtracted as described in Sec.II above. Conversion into the center-of-momentum (c.m.) system was performed individually for each bin of the histogram by a computer code which takes into account the geometry of the experiment, the integrated number of accumulated ${ }^{8} \mathrm{~B}$ ions, the energy losses of the ${ }^{8} \mathrm{~B}$ and protons in the target, and the effective target thickness for a specific bin. Two major features in the excitation function, indicated by ellipses and labeled by letters $\mathrm{A}$ and $\mathrm{B}$, are apparent in Fig. 4. These features could have been associated with broad resonances at excitation energies $\sim 2.7$ $\mathrm{MeV}$ and $\sim 4 \mathrm{MeV}$ in ${ }^{9} \mathrm{C}$, but no states were reported or predicted in ${ }^{9} \mathrm{C}$ at these excitation energies [12]. The only known excited state in ${ }^{9} \mathrm{C}$ is a narrow $(100 \mathrm{keV})$ $1 / 2^{-}$state at $2.2 \mathrm{MeV}$ which cannot be associated with either of these features.

More information is available on the level structure of the mirror nucleus ${ }^{9} \mathrm{Li}$. The second excited state in ${ }^{9} \mathrm{Li}$, which has a tentative spin-parity assignment of $5 / 2^{-}$, has been observed at an excitation energy of $4.3 \mathrm{MeV}$ [12]. The analog state is a good candidate for an explanation of the second peak in the ${ }^{8} \mathrm{~B}+\mathrm{p}$ excitation function (feature $\mathrm{B}$ in Fig. 4). However, the first peak (feature A) has no suitable counterpart in the spectrum of ${ }^{9} \mathrm{Li}$. The large width of this peak $(\sim 500 \mathrm{keV})$ at $1.3 \mathrm{MeV}$ above the threshold for proton decay indicates that the wave function of this state should have a significant contribution from the single particle configuration $\psi_{p} \otimes \psi_{8_{B}(g s)}$. A state with such properties should have been observed in the recent ${ }^{8} \mathrm{Li}(\mathrm{d}, \mathrm{p})$ experiment of Wuosmaa, et al., yet no states between the known $1 / 2^{-}$state at $2.6 \mathrm{MeV}$ and

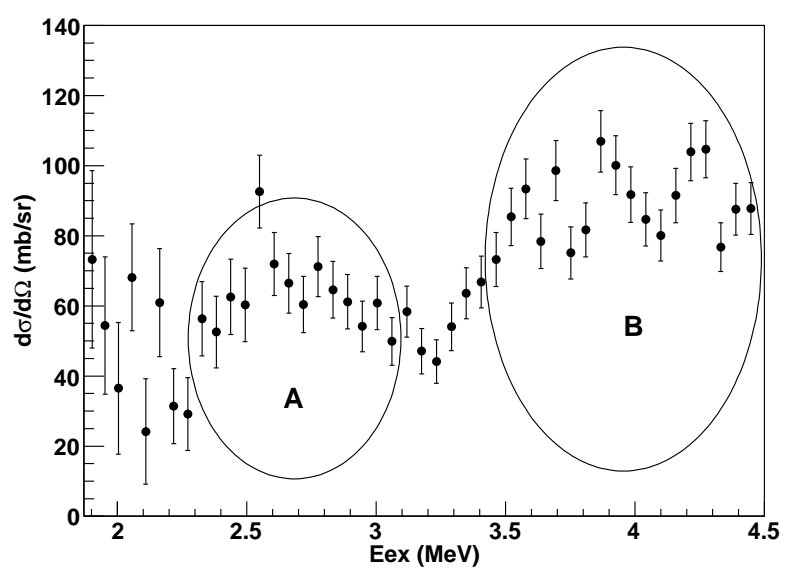

Figure 4: Excitation function for resonance scattering of ${ }^{8} \mathrm{~B}$ on protons at $164^{\circ} \pm 7^{\circ}$. The ${ }^{9} \mathrm{C}$ excitation energy is shown on the $\mathrm{x}$-axis (this is the c.m. energy plus the proton binding energy of $1.3 \mathrm{MeV}$ ).

the state at $4.3 \mathrm{MeV}$ were observed [13].

Various theoretical calculations also fail to offer any hint on what the structure at $2.7 \mathrm{MeV}$ might be. No states in ${ }^{9} \mathrm{Li} /{ }^{9} \mathrm{C}$ between the $1 / 2^{-}$and $5 / 2^{-}$states are predicted, either by large basis no-core shell-model calculations [2], or by Quantum Monte Carlo calculations [1]. (These predictions have to be treated with caution since configurations that allow positive parity states were not included). Continuum Shell Model (CSM) calculations, described in the previous section, also predict no states between the $1 / 2^{-}$and the $5 / 2^{-}$states. The considerations discussed above do not completely rule out a state at $2.7 \mathrm{MeV}$ in ${ }^{9} \mathrm{C}$, but they make its existence very unlikely and stimulate the search for another explanation of this feature.

The main disadvantage of the experimental technique used in this experiment is the inability to distinguish between elastic and inelastic scattering. Indeed, in the case of inelastic scattering, the recoil proton produced in the process also can hit the detector and hence be mistaken for an elastically-scattered proton. Normally, at low excitation energies, the cross section for resonance elastic scattering is much higher than that for inelastic scattering and the contribution from inelastic processes can safely be ignored. In fact, this is almost the case here. Estimations made using both CSM and R-matrix approaches (details are given in the following section) produce a cross section for the inelastic process which is one order of magnitude lower than that for elastic scattering. However, since the excitation energy of the first excited state of ${ }^{8} \mathrm{~B}$ is $0.77 \mathrm{MeV}$, which is $0.63 \mathrm{MeV}$ above the threshold of the ${ }^{8} \mathrm{~B}$ proton decay, an extra proton will be produced in the inelastic scattering process. Moreover, due to the inverse kinematics of the experiment, the extra proton will be focused more toward forward angles, increasing the chance to hit the detectors. The 
Table I: Continuum Shell Model results for ${ }^{9} \mathrm{C}$ performed with the WBP interaction 10] in an s-p-sd-pf valence space. Calculated excitation energies, widths, spectroscopic factors and eigenstate reorientation angles are given for the first five states in ${ }^{9} \mathrm{C}$. The known experimental value for the excitation energy of the $1 / 2^{-}$state [12] and the excitation energy of the $5 / 2^{-}$state measured in this work were used in the reaction calculations.

\begin{tabular}{ccccc|ccc|ccc}
\hline & $\mathrm{E}_{t h}$ & $\mathrm{E}_{\text {exp }}$ & $\Gamma$ & $\Theta$ & \multicolumn{3}{c|}{$\psi_{p} \otimes \psi_{8_{B}(g s)}$} & \multicolumn{3}{c}{$\psi_{p} \otimes \psi_{8_{B}(1+)}$} \\
$\mathrm{J}^{\pi}$ & $(\mathrm{MeV})$ & $(\mathrm{MeV})$ & $(\mathrm{MeV})$ & $\mathrm{deg}$ & $\mathrm{S}\left(\mathrm{p}_{3 / 2}\right)$ & $\mathrm{S}\left(\mathrm{p}_{1 / 2}\right)$ & $\Gamma_{W S}$ & $\mathrm{~S}\left(\mathrm{p}_{3 / 2}\right)$ & $\mathrm{S}\left(\mathrm{p}_{1 / 2}\right)$ & $\Gamma_{W S}$ \\
\hline $3 / 2^{-}$ & 0.00 & 0.00 & 0.000 & 0.0 & 0.87 & 0.00 & 0.0 & 0.18 & 0.00 & 0.0 \\
$1 / 2^{-}$ & 1.4 & 2.2 & 0.027 & 0.1 & 0.18 & 0.00 & 0.15 & 0.75 & 0.00 & $7.6 \mathrm{E}-3$ \\
$5 / 2^{-}$ & 3.9 & 3.6 & 1.30 & 5.1 & 0.13 & 0.59 & 1.80 & 0.00 & 0.00 & 0.59 \\
$3 / 2^{-}$ & 4.1 & - & 1.32 & 10.8 & 0.08 & 0.09 & 3.19 & 0.23 & 0.47 & 1.14 \\
\hline & & & & $\mathrm{S}\left(\mathrm{d}_{5 / 2}\right)$ & $\mathrm{S}\left(\mathrm{d}_{3 / 2}\right)$ & $\mathrm{S}\left(\mathrm{s}_{1 / 2}\right)$ & $\mathrm{S}\left(\mathrm{d}_{5 / 2}\right)$ & $\mathrm{S}\left(\mathrm{d}_{3 / 2}\right)$ & $\mathrm{S}\left(\mathrm{s}_{1 / 2}\right)$ & \\
\hline $3 / 2^{+}$ & 4.2 & - & 2.1 & 16.0 & 0.00 & 0.01 & 0.41 & 0.05 & 0.002 & 0.03 \\
\hline
\end{tabular}

Table II: Continuum Shell Model results for ${ }^{9} \mathrm{C}$ performed with the PWT interaction [10] in a p-shell-only valence space.

\begin{tabular}{ccc|ccc|ccc}
\hline & $\mathrm{E}_{x}$ & $\Gamma$ & \multicolumn{3}{|c|}{$\psi_{p} \otimes \psi_{8_{B}(g s)}$} & \multicolumn{3}{|c}{$\psi_{p} \otimes \psi_{8_{B}(1+)}$} \\
$\mathrm{J}^{\pi}$ & $(\mathrm{MeV})$ & $(\mathrm{MeV})$ & $\mathrm{S}\left(\mathrm{p}_{3 / 2}\right)$ & $\mathrm{S}\left(\mathrm{p}_{1 / 2}\right)$ & $\mathrm{S}(\mathrm{tot})$ & $\mathrm{S}\left(\mathrm{p}_{3 / 2}\right)$ & $\mathrm{S}\left(\mathrm{p}_{1 / 2}\right)$ & $\mathrm{S}($ tot $)$ \\
\hline $3 / 2^{-}$ & 0.00 & 0.000 & 0.95 & 0.02 & 0.97 & 0.34 & 0.03 & 0.37 \\
$1 / 2^{-}$ & 2.2 & 0.045 & 0.30 & 0.0 & 0.30 & 0.49 & 0.01 & 0.50 \\
$5 / 2^{-}$ & 3.6 & 1.44 & 0.11 & 0.68 & 0.79 & 0.03 & 0.00 & 0.03 \\
$3 / 2^{-}$ & 4.1 & 1.25 & 0.01 & 0.16 & 0.17 & 0.24 & 0.38 & 0.62 \\
\hline
\end{tabular}

energy of this proton can be estimated using the following arguments. The cross section for inelastic scattering is likely to increase toward higher beam energies due to higher penetrability factors. Hence most of the "inelastic" protons are produced at the beginning of the target. After inelastic scattering, the ${ }^{8} \mathrm{~B}$ recoils are focused into a narrow cone $\left(\sim 6^{\circ}\right.$ with respect to the beam axis $)$ and have an energy of $\sim 3.0 \mathrm{MeV} / \mathrm{A}$. The energy of protons produced in the decay of the excited state of ${ }^{8} \mathrm{~B}$ is:

$$
E_{p}=\frac{E_{8} B}{8}+\frac{7}{8} Q+2 \sqrt{\frac{7}{8}} Q \sqrt{\frac{E_{8} B}{8}} \cos \theta
$$

where $\mathrm{Q}$ is the decay energy $(0.63 \mathrm{MeV})$ and $\theta$ is the angle between the momentum vectors of the proton and the ${ }^{8} \mathrm{~B}$, which has to be close to $0^{\circ}$ or $180^{\circ}$ in order for the proton to hit the detector at $7^{\circ}$. If $\theta$ is $0^{\circ}$, then $\mathrm{E}_{p}$ is roughly $6 \mathrm{MeV}$. The energy loss of $6 \mathrm{MeV}$ protons in the $9 \mathrm{mg} / \mathrm{cm}^{2}$ plastic target is $\sim 1 \mathrm{MeV}$. This leaves us with $5 \mathrm{MeV}$ protons in the detector. Note that only a limited range of angles $\theta$ can produce a hit in the detector. Hence the protons from the decay of the first excited state of ${ }^{8} \mathrm{~B}$ will peak at around $5 \mathrm{MeV}$. This is exactly the energy of the first peak (A) in the laboratory frame (see Fig. 3). On the other hand, if the angle $\theta$ is close to $180^{\circ}$ the proton energy will be $\sim 1.0 \mathrm{MeV}$ and these protons will be stopped in the target. Therefore, only one peak from the proton decay of the ${ }^{8} \mathrm{~B}$ first excited state can be observed.

A realistic Monte-Carlo simulation of the inelastic scattering ${ }^{1} \mathrm{H}\left({ }^{8} \mathrm{~B}, \mathrm{p}^{\prime}\right){ }^{8} \mathrm{~B}^{*}\left(1^{+} ; 0.77 \mathrm{MeV}\right)$ with subsequent proton decay of ${ }^{8} \mathrm{~B}$ was performed. The simulation took into account the specific geometry of the experiment, the kinematics of the process, energy losses of the ${ }^{8} \mathrm{~B}$ and protons in the target, energy straggling, and multiple scattering (the GEANT 3.21 package was used [14]). The result of this simulation is shown in Figure 5. The first peak of the shaded histogram in Fig. 5] is associated with protons from the decay of the excited ${ }^{8} \mathrm{~B}$ state, and the second peak corresponds to the recoil protons inelastically scattered by ${ }^{8} \mathrm{~B}$. It can be seen from Fig. [5 that "inelastic" protons can entirely account for the peak observed in the proton spectrum at $5 \mathrm{MeV}$ if the cross section for inelastic scattering of ${ }^{8} \mathrm{~B}$ is $\sim 1 / 10$ of the elastic cross section at 29 $\mathrm{MeV}$. In the following analysis, we will assume that this is the case and subtract the "inelastic" proton contribution from the excitation function.

\section{R-MATRIX ANALYSIS}

The excitation function of resonance elastic scattering of ${ }^{8} \mathrm{~B}$ on protons resulting from the subtraction of "direct" proton background, "carbon" background and assumed "inelastic" proton background, and converted into the c.m. system, is shown in Figure[6] A two-channel multilevel R-matrix approach was then applied for the analysis of the excitation function. Besides the elastic channel, the first inelastic channel was included in the R-matrix calculation. In order to make the R-matrix fit more realistic, we start by deducing the reduced-width amplitudes from the CSM spectroscopic amplitudes. Since these amplitudes have been calculated in the jj-coupling scheme, they must be re-coupled into LS coupling as used in the 


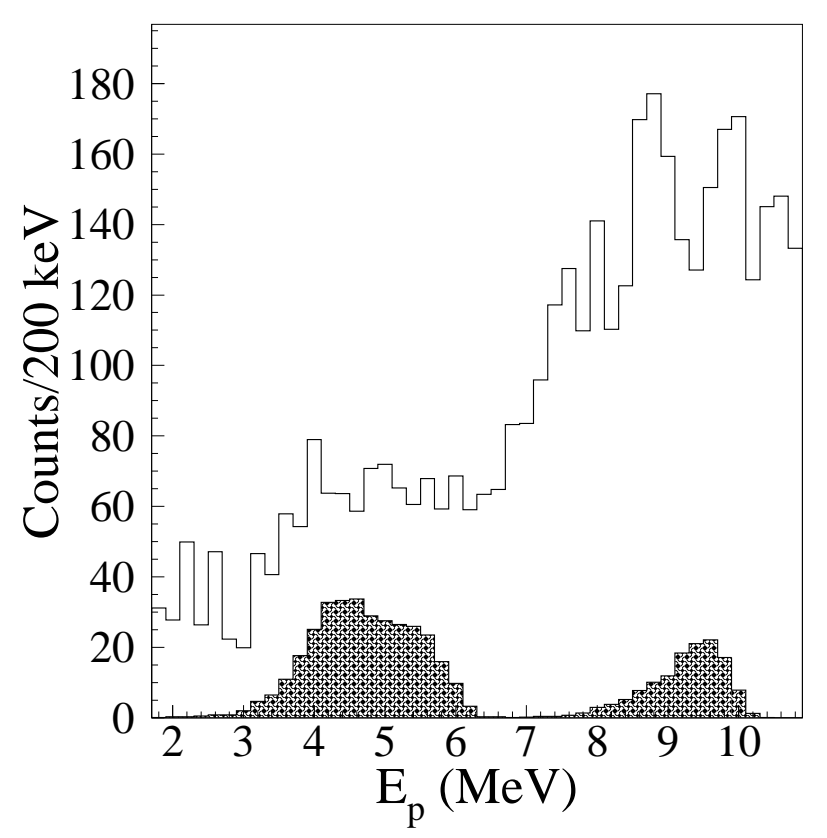

Figure 5: The proton spectrum in the laboratory system with the carbon background subtracted. The Monte-Carlo simulation of the inelastic process ${ }^{1} \mathrm{H}\left({ }^{8} \mathrm{~B}, \mathrm{p}^{\prime}\right){ }^{8} \mathrm{~B}^{*}\left(1^{+} ; 0.77 \mathrm{MeV}\right)$ is shown as a shaded histogram. The two peaks at 5 and $9 \mathrm{MeV}$ in the shaded histogram are associated with the proton decay of the ${ }^{8} \mathrm{~B}$ excited state and the inelastically scattered proton, respectively.

R-matrix approach. This can be accomplished using the $6 j$-symbols, according to the following expression:

$$
\begin{aligned}
\gamma_{\lambda c}= & \left(\frac{\hbar^{2}}{\mu_{c} a_{c}^{2}}\right)^{\frac{1}{2}} \sum_{j} A_{\lambda c j}(-1)^{I_{1}+I_{2}+\ell+J} \\
& \sqrt{2 S+1} \sqrt{2 j+1}\left\{\begin{array}{ccc}
I_{1} & I_{2} & S \\
\ell & J & j
\end{array}\right\},
\end{aligned}
$$

where $c$ corresponds to a specific channel with a set of quantum numbers $\mathrm{J}, \ell, \mathrm{S}$ (the channel spin $S=I_{1}+$ $\left.I_{2}\right), I_{1}$ (the spin of the projectile), $I_{2}$ (the spin of the target), $\mu_{c}$ is the reduced mass, $a_{c}$ is the channel radius $(4.5 \mathrm{fm})$, and $A_{\lambda c j}$ is the CSM spectroscopic amplitude (the relative phase was calculated in the CSM and defined by the sign of the $\left.A_{\lambda c j}\right)$. The sum is taken over the relevant single-particle orbits $(j=1 / 2$ and $3 / 2$ for $\mathrm{p}$ shell states). Reduced-width amplitudes, calculated as described above, were then varied about the calculated values to obtain the best fit to the data shown in Fig. [6]

It is clear that the known $1 / 2^{-}$first excited state of ${ }^{9} \mathrm{C}$, at an excitation energy of $2.2 \mathrm{MeV}$ and having a width of $100 \mathrm{keV}$ [12], cannot account for the large cross section observed at higher energy (dotted curve in Fig 6). Introduction of a broad $5 / 2^{-}$state at an excitation energy of $\sim 3.6 \mathrm{MeV}$ produces a reasonable agreement between the R-matrix calculation and the experimental data (solid

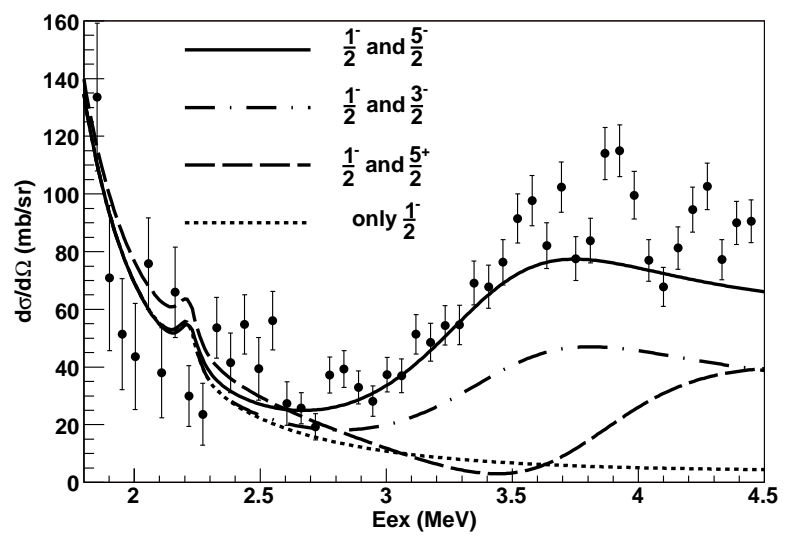

Figure 6: The excitation function of resonance elastic scattering of ${ }^{8} \mathrm{~B}$ on protons after subtraction of "direct", "carbon" and "inelastic" backgrounds. The solid curve is an R-matrix fit with the known $1 / 2^{-}$resonance plus a $5 / 2^{-}$state at an excitation energy of $3.6 \mathrm{MeV}$ having a width of $1.5 \mathrm{MeV}$. The dotted curve shows the contribution of the $1 / 2^{-}$state alone. $\mathrm{R}$-matrix calculations for the $1 / 2^{-}$state together with $3 / 2^{-}$ or $5 / 2^{+}$states are shown as dash-dotted and dashed curves, respectively.

curve in Fig. 6). The excitation energy and width of the assumed $5 / 2^{-}$state are $3.6 \pm 0.2 \mathrm{MeV}$ and $1.4 \pm 0.5$ $\mathrm{MeV}$, respectively. No other spin-parity assignment for the state at $3.6 \mathrm{MeV}$ agrees with the experimental data. The cross section for the $3 / 2^{-}$state is too small (dashdotted curve in Fig. 6), and the positive parity states, with $\ell=0$ dominant partial wave, produce a dip in the excitation function due to destructive interference (dashed curve in Fig. (6).

Two problems can be identified in the description of the excitation function as a combination of only two states, $1 / 2^{-}$and $5 / 2^{-}$. First, the measured cross section is still somewhat higher at $\sim 4 \mathrm{MeV}$ than can be accounted for by the $5 / 2^{-}$state in ${ }^{9} \mathrm{C}$. Second, the $5 / 2^{-}$ state is essentially a single-particle state and decays predominantly to the ground state of ${ }^{8} \mathrm{~B}$. If no other states are introduced, the cross section for inelastic scattering $\left[{ }^{1} \mathrm{H}\left({ }^{8} \mathrm{~B}, \mathrm{p}^{\prime}\right)^{8} \mathrm{~B}\left(1^{+}\right)\right]$would be too small to account for the "inelastic" peak at $5 \mathrm{MeV}$ in the laboratory proton spectrum (Fig. [5). Both problems can be resolved at once if an additional state with substantial contribution from the ${ }^{8} \mathrm{~B}\left(1^{+}\right)+\mathrm{p}$ configuration is introduced. The CSM calculations predict two states in close proximity to the $5 / 2^{-}$ state (see Table I). These are $3 / 2^{-}$and $3 / 2^{+}$states at 4.1 and $4.2 \mathrm{MeV}$. The wave function of the $3 / 2^{-}$state has a large inelastic component (Table 【). Hence, the introduction of this state can potentially fix both problems by increasing the cross section at $\sim 4 \mathrm{MeV}$ and explaining the observed "inelastic" peak. The solid curve in Fig. 7 shows an R-matrix fit with $1 / 2^{-}, 5 / 2^{-}$and $3 / 2^{-}$states. The dashed curve in Fig. 7 represents the inelastic excitation function due to the $3 / 2^{-}$state. 
Table III: Excitation energies, energy eigenvalues, widths and reduced-width amplitudes of the resonances deduced in ${ }^{9} \mathrm{C}$.

$\ell \mathrm{S} \quad \mathrm{B}_{c}$

\begin{tabular}{|c|c|c|c|c|}
\hline $\mathrm{J}^{\pi}$ & & $\frac{1}{2}^{-}$ & $\frac{5}{2}^{-}$ & $\frac{3}{2}^{-}$ \\
\hline $\mathrm{E}_{e x}(\mathrm{MeV})$ & & 2.22 & 3.6 & 4.1 \\
\hline $\mathrm{E}_{\lambda}(\mathrm{MeV})$ & & 0.61 & 3.45 & 4.15 \\
\hline$\Gamma(\mathrm{MeV})$ & & 0.10 & 1.4 & 1.3 \\
\hline \multirow{3}{*}{$p+{ }^{8} B$ (g.s.) } & $1 \frac{3}{2}-1.2$ & $1.15(0.65)$ & $0.33(0.47)$ & $0.17(0.17)$ \\
\hline & $1 \frac{5}{2}-1.2$ & - & $-1.34(-1.20)$ & $0.59(0.59)$ \\
\hline & $1 \frac{1}{2}-1.2$ & $1.25(1.25)$ & & $-0.15(-0.15)$ \\
\hline $\mathrm{p}+{ }^{8} \mathrm{~B}\left(1^{+}\right)$ & $1 \frac{3}{2}-1.2$ & $0.42(0.42)$ & $0.00(0.00)$ & $1.27(1.27)$ \\
\hline
\end{tabular}

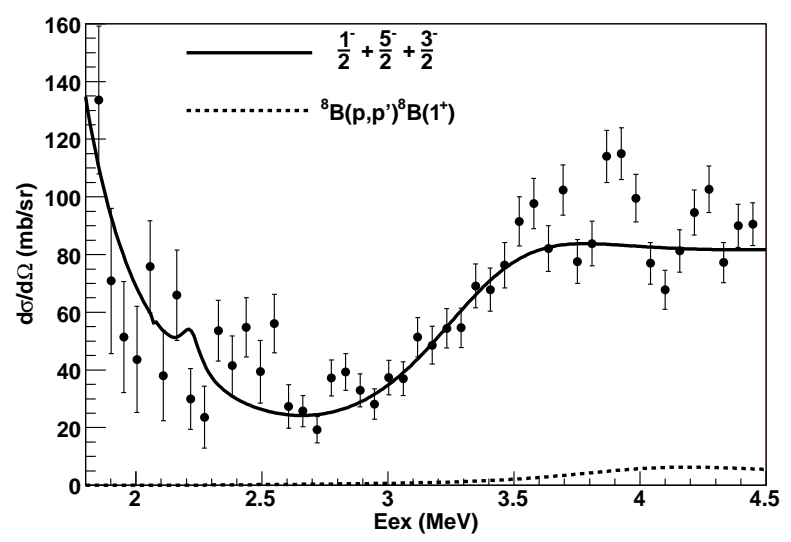

Figure 7: Solid curve is an R-matrix fit of the ${ }^{8} \mathrm{~B}+\mathrm{p}$ excitation function which includes $1 / 2^{-}, 5 / 2^{-}$and $3 / 2^{-}$resonances. Dotted curve is the calculated excitation function of the resonance inelastic scattering (it is mostly due to the $3 / 2^{-}$ resonance).

The best-fit R-matrix parameters for all three states are shown in Table III (the values given in parenthesis are reduced-width amplitudes calculated from CSM spectroscopic amplitudes using the WBP [10] interaction, according to the expression 21). The parameters for the $1 / 2^{-}$state (energy eigenvalue and elastic reduced-width amplitude) were adjusted to reproduce the experimental excitation energy and width [12]. The parameters for the $3 / 2^{-}$state were derived from CSM and were not varied. As follows from the Table III no significant modifications of the calculated CSM reduced-width amplitudes (except for the elastic reduced-width amplitude of the $1 / 2^{-}$state) were necessary to fit the experimental data.

\section{DISCUSSION}

The main result of this work is a firm identification of the $5 / 2^{-}$resonance in ${ }^{9} \mathrm{C}$. Its excitation energy is $3.6 \pm 0.2$ $\mathrm{MeV}$ and its width is $1.4 \pm 0.5 \mathrm{MeV}$. This state is a feature of all theoretical calculations, ab initio [1, 2] and shell-model alike, which seem to agree on the structure and excitation energy of the state. These predict that it should be in the vicinity of $3.5 \mathrm{MeV}$ and have a single particle structure with spectroscopic factor of about 0.8 . The experimental width of the $5 / 2^{-}$state is in very good agreement with the CSM width $(1.30 \mathrm{MeV})$. Based on this comparison, the experimental single-particle spectroscopic factor of the $5 / 2^{-}$state is $0.77 \pm 0.25$. This result is also in very good agreement with the recent experimental result of Wuosmaa, et al. [13], in which the spectroscopic factor of the mirror state in ${ }^{9} \mathrm{Li}$ was measured to be $0.93 \pm 0.2$ using the ${ }^{8} \mathrm{Li}(\mathrm{d}, \mathrm{p})$ reaction.

Unfortunately, the statistics in the present experiment were not sufficient to observe the weak $1 / 2^{-}$first excited state. The direct observation of this state in the ${ }^{8} \mathrm{~B}+\mathrm{p}$ excitation function would allow for an accurate measurement of its width. At the moment, it seems that theoretical calculations (especially shell model) tend to underestimate the single particle spectroscopic factor of this state. The width of this state in ${ }^{9} \mathrm{C}$ was determined in a single, low-statistics experiment using the ${ }^{12} \mathrm{C}\left({ }^{3} \mathrm{He},{ }^{6} \mathrm{He}\right)$ reaction [6], and the measured value of $100 \pm 20 \mathrm{keV}$ indicates that the spectroscopic factor of the state is $\sim 0.67 \pm 0.15$ based on a comparison to the CSM width given in Table@ A similar result was obtained for the spectroscopic factor of the $1 / 2^{-}$state in ${ }^{9} \mathrm{Li}$ using the ${ }^{8} \mathrm{Li}(\mathrm{d}, \mathrm{p})$ reaction [13]. However, the theoretical predictions for the spectroscopic factor (which range from 0.17 to 0.5 depending on the model and interaction used in the SM) are consistently lower than the experimental value. Hence, it would be of interest to verify the result of the previous experiment and improve the experimental accuracy for the value of the width of this state.

In addition to the known $1 / 2^{-}$state and the proposed new $5 / 2^{-}$state, the experimental data indicate the presence of an additional state or states at or above $4 \mathrm{MeV}$ with strong decay branch(es) into the first excited state of ${ }^{8} \mathrm{~B}$. The $3 / 2^{-}$state predicted by CSM at $4.1 \mathrm{MeV}$ (Table seems to be a good candidate. The inclusion of the $3 / 2^{-}$state improved the fit and provided for an explanation of the "inelastic" peak at $5 \mathrm{MeV}$ of proton laboratory energy. The inelastic cross section due to the $3 / 2^{-}$state (shown in Figure 7) was calculated using an R-matrix approach with parameters for the $3 / 2^{-}$state derived from the CSM as described in the previous section. This cross section can also be calculated directly in the CSM approach in which all the resonances (including resonances at higher excitation energy) are included automatically with correct interference. The comparison of the total ${ }^{8} \mathrm{~B}\left(\mathrm{p}, \mathrm{p}^{\prime}\right)^{8} \mathrm{~B}\left(1^{+}\right)$inelastic cross section, calculated using the R-matrix approach and the CSM, is shown in Fig. 8. The magnitude and the shape of the total inelastic cross section is similar in both calculations. This comparison is instructive in many ways. First, it shows that the R-matrix calculation with reduced-width amplitudes derived from the CSM spectroscopic amplitudes using Eq. 2 produces a cross section in the vicinity of the $3 / 2^{-}$resonance which is similar to the one calculated directly in 


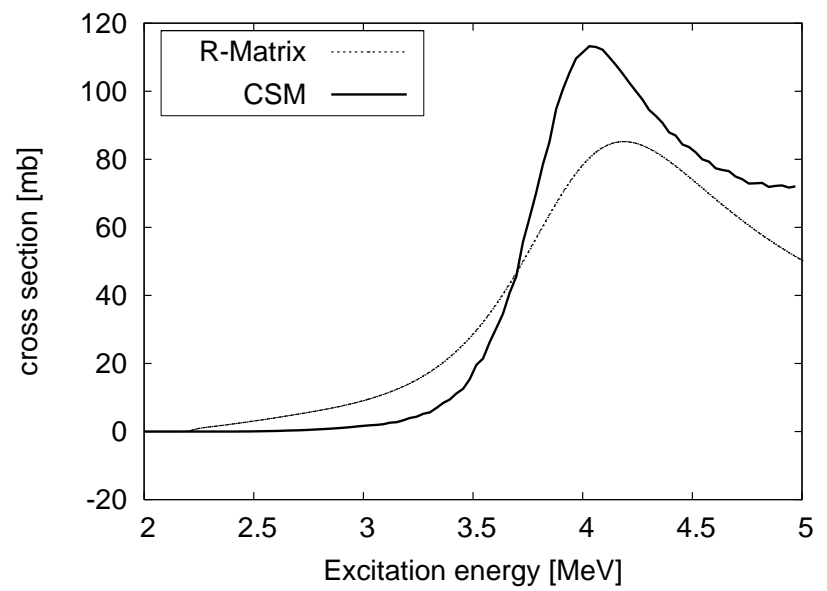

Figure 8: The total cross section of the inelastic scattering process ${ }^{8} \mathrm{~B}\left(\mathrm{p}, \mathrm{p}^{\prime}\right)^{8} \mathrm{~B}\left(1^{+}\right)$. The dotted curve was calculated using the $\mathrm{R}$-matrix approach with resonance parameters given in Table III The solid curve is the CSM calculation, which automatically take into account the influence of higher- lying resonances.

the CSM . Second, it indicates that, while the $3 / 2^{-}$resonance included in the R-matrix calculation determines the inelastic cross section at $\sim 4 \mathrm{MeV}$, the shape of the inelastic cross section is influenced by higher- lying resonances (especially by the next $3 / 2^{-}$state). The CSM automatically takes this into account, providing a realistic estimate of the influence of background resonances and accounting for the possible interference of this background with the main resonances. This feature of the CSM is important for future studies of the properties of exotic nuclei in resonance scattering. Unfortunately, due to the limited excitation energy range, relatively low statistics, and inability to separate elastic from inelastic processes, the inelastic cross section cannot be accurately extracted from the present experimental data and the actual parameters of the $3 / 2^{-}$state cannot be determined.

In Ref. [7], the authors claim that they observe a resonance in ${ }^{9} \mathrm{C}$ at $3.3 \mathrm{MeV}$ in the ${ }^{12} \mathrm{C}\left({ }^{3} \mathrm{He},{ }^{6} \mathrm{He}\right)$ reaction. Based on the assumption of mutual correspondence between the $4.3 \mathrm{MeV}$ state in ${ }^{9} \mathrm{Li}$ and the observed state in ${ }^{9} \mathrm{C}$, they concluded that the state cannot be $5 / 2^{-}$, arguing that the observed Thomas-Ehrman shift is too large for an $\ell=1$ state. A $5 / 2^{+}$spin-parity assignment was proposed instead. However, close examination of the ${ }^{6} \mathrm{He}$ spectrum in Fig. 1 of Ref. [7] reveals that, due to low statistics and poor experimental resolution $(\sim 500$ $\mathrm{keV}$ ), the spectrum allows for a different interpretation and also does not contradict assignment of a broad state at 3.6 MeV. Moreover, the value for the Thomas-Ehrman shift of the $5 / 2^{-}$state in ${ }^{9} \mathrm{Li} /{ }^{9} \mathrm{C}$ can be estimated from a simple potential model assuming a pure single-particle nature for this state. For example, a Woods-Saxon potential with common parameters $\left(r_{\circ}=1.21 \mathrm{fm}, a=0.65\right.$ $\mathrm{fm}$ ) and depth adjusted to reproduce the c.m. energy of the $4.3 \mathrm{MeV}$ state in ${ }^{9} \mathrm{Li}$ gives $0.5 \mathrm{MeV}$ for the ThomasEhrman shift of the $\ell=1$ state. This value is a factor of four bigger than the one assumed in Ref. [7] and suggests that the excitation energy of the $5 / 2^{-}$state in ${ }^{9} \mathrm{C}$ should be $\sim 3.8 \mathrm{MeV}$. This value is in very good agreement with the results of this work, lending further support to a $5 / 2^{-}$ spin-parity assignment for the resonance in question.

\section{CONCLUSION}

The excitation function for resonance scattering ${ }^{8} \mathrm{~B}+\mathrm{p}$ was measured using the thick-target inverse-kinematics technique. $\mathrm{A}{ }^{9} \mathrm{C}$ excitation energy range from $1.8 \mathrm{MeV}$ to $4.5 \mathrm{MeV}$ was covered. One new state in ${ }^{9} \mathrm{C}$ was identified at an excitation energy of $3.6 \pm 0.2 \mathrm{MeV}$, having a width of $1.4 \pm 0.5 \mathrm{MeV}$. R-matrix analysis of the excitation function allows for a unique $5 / 2^{-}$spin-parity assignment to this state. It has a single-particle nature with a spectroscopic factor of $0.77 \pm 0.25$, consistent with theoretical predictions of the $a b$ initio models and CSM calculations, and also with recent experimental results for the presumed mirror state in ${ }^{9} \mathrm{Li}[13$. The measured excitation function indicates the existence of higher-lying states with strong inelastic decay branches. A new measurement with higher statistics, broader excitation energy and angular range coverage is highly desirable. The new experiment must be designed in a way that allows for separation of elastic and inelastic scattering.

Using the CSM as a part of the analysis, we attempted to take a step beyond the typical perturbative approach based on spectroscopic factors and R-matrix analysis. In the case of the newly-discovered resonances, due to their isolated nature, the role of the continuum appears to be reasonably well described by perturbation theory. However, the onset of physics that demands the use of a unified structure-reaction approach is clearly indicated. As an example, the experimental analysis hinges on tracking the contribution from an inelastic channel that appears to be dominated by overlapping $3 / 2^{-}$resonances. The CSM was used to compute the cross section for this process, and comparison with the R-matrix fit indicates a nontrivial nature for new physics on the structure/reaction border that is successfully captured by the novel CSM technique.

\section{Acknowledgments}

The authors are grateful to Prof. Goldberg for helpful discussions. This work was supported by the National Science Foundation under Grant Nos. PHY04-56463 and PHY03-54828, and by U.S. Department of Energy Contract No. DE-FG02-92ER40750. 
[1] S. C. Pieper, K. Varga, and R. B. Wiringa, Phys. Rev. C 66, 044310 (2002).

[2] P. Navratil and B. R. Barrett, Phys. Rev. C 57, 3119 (1998).

[3] N. Michel, W. Nazarewicz, M. Ploszajczak, and J. Okolowicz, Phys. Rev. C 67, 054311 (2003).

[4] A. Volya and V. Zelevinsky, Phys. Rev. Lett. 94, 052501 (2005).

[5] J. Cerny, R. H. Pehl, F. S. Goulding, and D. A. Landis, Phys. Rev. Lett. 13, 726 (1964).

[6] W. Benenson and E. Kashy, Phys. Rev. C 10, 2633 (1974).

[7] M. S. Golovkov, V. Z. Goldberg, L. S. Danelyan, V. I. Dukhanov, I. L. Kuleshov, A. E. Pakhomov, I. N. Serikov, V. A. Timofeev, and V. N. Unezhev, Sov. J. Nucl. Phys. 53, 550 (1991).

[8] M. Y. Lee, F. D. Becchetti, T. W. O'Donnell, D. A. Roberts, J. A. Zimmerman, V. Guimaraes, J. J. Kolata, D. Peterson, P. Santi, P. A. DeYoung, et al., Nucl. In- strum. Methods Phys. Res., Sect. A 422, 536 (1999).

[9] K. P. Artemov, O. P. Belyanin, A. L. Vetoshkin, R. Wolski, M. S. Golovkov, V. Z. Goldberg, M. Madeja, V. V. Pankratov, I. N. Serikov, V. A. Timoveef, et al., Sov. J. Nucl. Phys. 52, 408 (1990).

[10] B. A. Brown, Prog. Part. Nucl. Phys. 47, 517 (2001).

[11] N. Schwierz, I. Wiedenhover, and A. Volya, submitted to Phys. Rev. C.

[12] D. R. Tilley, J. H. Kelley, J. L. Godwin, D. J. Millener, J. E. Purcell, C. G. Sheu, and H. R. Weller, Nucl. Phys. A 745, 155 (2004).

[13] A. H. Wuosmaa, K. E. Rehm, J. P. Greene, D. J. Henderson, R. V. F. Janssens, C. L. Jiang, L. Jisonna, E. F. Moore, R. C. Pardo, M. Paul, et al., Phys. Rev. Lett. 94, 082502 (2005).

[14] R. Brun et al., CERN Program Library Long Write-up, CERN W5013 (1994). 